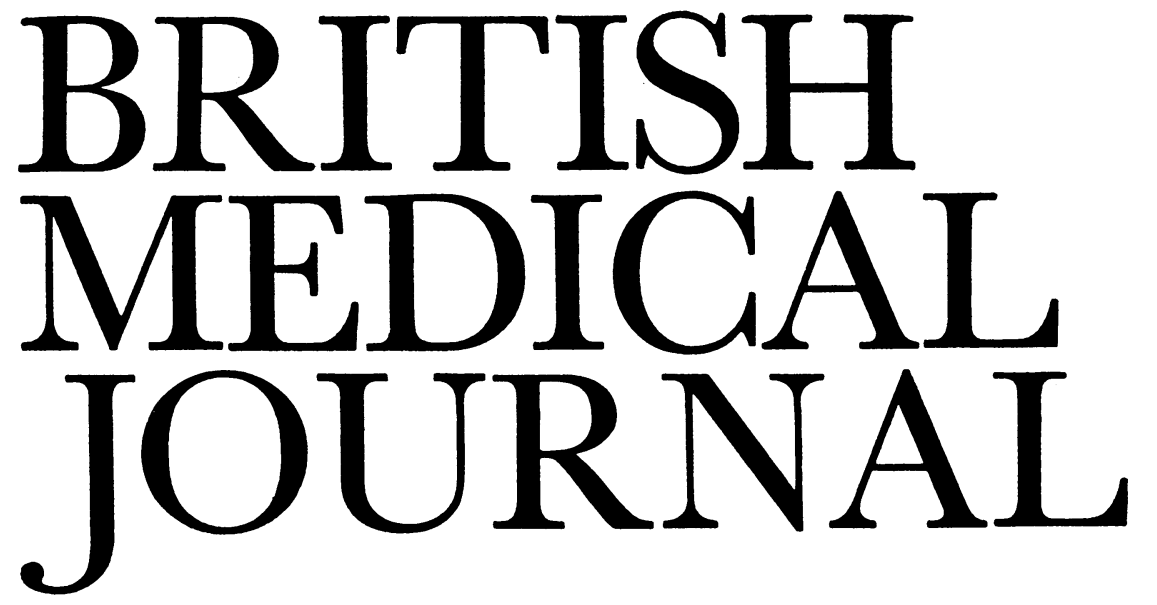

\title{
Tuberculous Meningitis in Children
}

Tuberculous meningitis has become relatively rare in countries with advanced social and medical standards. With modern treatment almost $100 \%$ recovery can be achieved without sequelae if it is begun before the child becomes unconscious. ${ }^{1}$ But among underdeveloped peoples the disease is of much graver import. For example, in a recent South African series I. Freiman and J. Geef huysen ${ }^{2}$ treated 131 African children of whom two-thirds were unconscious at the beginning of therapy. About $40 \%$ of all the patients had died within a year, and more than half the survivors had serious sequelae.

Early diagnosis makes all the difference in this disease. Listlessness, irritability, anorexia and vomiting, loss of weight, refusal to smile or play, or photophobia may all indicate the onset of tuberculous meningitis in an infant or child. Older children will complain of headache. The persistence and the combination of several of these symptoms should alert the doctor to the possibility of tuberculous meningitis before the appearance of neck stiffness, focal neurological signs, disturbance of consciousness, or convulsions. A positive contact history is important but is now rarely obtained. More often the discovery of tuberculosis in a child followed by an immediate search will lead to the detection of an unsuspected active case in the household, or among relatives or friends, or in the school. The discovery of such a contact is a useful diagnostic aid.

An intradermal tuberculin test (Mantoux 1:1,000) should be carried out at once, but in advanced cases a negative result does not rule out tuberculous meningitis. Tuberculin testing of all children should still be a routine in hospitals, for to omit it may lead to delay in diagnosis. Choroidal tubercles are present at the onset in about half the cases ${ }^{3}{ }^{4}$ and provide an immediate diagnostic clue. They are easily found in a wellsedated child whose pupils are fully dilated. A radiograph of the chest may show hilar glands, a primary complex, or miliary tuberculosis. In suspected cases at least $10 \mathrm{ml}$ of cerebrospinal fluid should be obtained and the deposit examined for tubercle bacilli. These can be detected in the overwhelming majority of cases without difficulty in a skilled laboratory. This is much less possible if too small samples are provided. A clear cerebrospinal fluid with pleocytosis in which lymphocytes predominate and in which the sugar level is low and the protein is raised usually indicates tuberculous menin- gitis, but similar fluid may be found in viral meningitis or in bacterial meningitis after partial antibiotic treatment. To settle the diagnosis a second large sample of cerebrospinal fluid should be examined without delay for the presence of tubercle bacilli on a smear, by culture, and by guinea-pig inoculation. Treatment should not be delayed further even in the absence of a positive smear if other evidence of tuberculosis is available at this stage.

The generally accepted antibacterial regimen consists of a combination of isoniazid ( $20 \mathrm{mg}$ per $\mathrm{kg}$ body weight daily) with streptomycin $(40 \mathrm{mg} / \mathrm{kg}$ ) and possibly with paraaminosalicylic acid $(250 \mathrm{mg} / \mathrm{kg})$ given systemically. There is no general agreement on the use of intrathecal therapy or corticosteroids. The value of several newer antituberculous drugs in the treatment of tuberculous meningitis remains uncertain. Among these rifampicin appears to be the most promising. In vitro it is as efficient as isoniazid, ${ }^{5}$ and it is useful against isoniazid-resistant organisms. Good concentrations may be achieved in the cerebrospinal fluid after oral administration. ${ }^{6}$ But unfortunately rifampicin is hepatotoxic and is very expensive, so its use is limited to specially difficult cases.

The place for intrathecal antibacterial therapy has not been determined by adequate controlled therapeutic trials. Many workers, particularly in the U.S.A., ${ }^{7} 8$ do not recommend giving streptomycin (or other drugs) by this route. It is true that hundreds of patients have survived without intrathecal therapy. But there are also many failures with this policy, and patients may die unnecessarily or suffer from preventable, permanent neurological sequelae. ${ }^{9} 10$ The best results have been obtained when the intrathecal therapy was given as a routine. ${ }^{1911}$ Clinical and bacteriological relapse is frequently seen after the cessation of intrathecal treatment while the patient is on full systemic therapy, including isoniazid. It can be successfully treated by further intrathecal injections of streptomycin or isoniazid. ${ }^{1}$ Patients not receiving initial intrathecal therapy often deteriorate seriously during systemic therapy, and the introduction of intensive intrathecal therapy can reverse the downhill progress even in desperate cases. ${ }^{10}$

Corticosteroids are often given systemically as adjuvants, but there is no proof that they are of value, except perhaps for the initial treatment of patients with severe cerebral oedema 
or with toxaemia due to massive miliary tuberculosis. ${ }^{10}$ Intrathecal hydrocortisone is effective in resolving impending spinal block and may dissolve existing blocks. The dosage recommended is $10 \mathrm{mg}$ with each intrathecal injection of isoniazid or streptomycin. Routine treatment with systemic or intrathecal corticosteroids is not recommended. ${ }^{1910}$ Freiman and Geefhuysen ${ }^{2}$ carried out a controlled trial under difficult conditions to determine the value of intrathecal streptomycin and hydrocortisone therapy, and though their study did not prove conclusively the value of intrathecal therapy it added to the existing evidence that its use is associated with better results.

Today we must expect recovery without sequelae in almost all patients who are conscious on admission as well as in many of the others. As intrathecal therapy is essential in some cases it is wise to give it initially to all patients, the routine course being 10 injections of streptomycin (10-50 mg per dose according to age). But longer or repeated courses should be given if necessary. Isoniazid (10-50 mg per dose according to age) and hydrocortisone (10-20 mg per dose) should be added in special circumstances, especially during relapse, or if there is a suspicion that the organism is getting less sensitive, or if spinal block is threatened. ${ }^{1}$ In late relapses or if there is no response to standard treatment the use of the new antituberculous drugs should be considered, especially rifampicin. ${ }^{6}$

In advanced cases much of the neurological damage and the intellectual retardation is due to the prolonged, high intracranial pressure leading to obstructive hydrocephalus. ${ }^{\mathbf{1 2}}$ This hydrocephalus can be relieved surgically while the child is still under systemic antituberculous therapy. All this implies long and formidable treatment, but the results justify it.

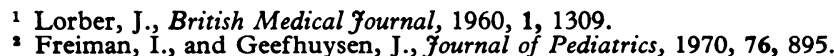

3 Illingworth, R. S., and W right, T., British Medical fournal, 1948, 2, 365.

- Illingworth, R. S., and Lorber, J., Archives of Disease in Childhood, 1956, $31,467$.

5 Canetti, G., le Lirzin, M., Porven, G., Rist, N., and Grumbach, F., Tubercle, 1968, 49, 367.

- Lorber, J., unpublished data.

Lincoln, E. M., Sordillo, S. R., and Davies, P. A., fournal of Pediatrics, 1960, 57, 807 .

Weiss, W., and Flippin, H. F., American fournal of the Medical Sciences, $1965,250,46$.

- Fitzsimmons, J. M., and Smith, H. M. V., Tubercle, 1963, 44, 103.

10 Hockaday, J. M., and Smith, H. M. V., Tubercle, 1966, 47, 75.

11 Miller, F. J. W., Seal, R. M. E., and Taylor, M. D., in Tuberculosis in Children, p, 416 . London, Churchill, 1963.

12 Lorber, J., Archives of Disease in Childhood, 1951, 26, 28.

\section{Platelet Transfusions}

The treatment of haemorrhage resulting from thrombocytopenia by the transfusion of fresh platelets is well established. But until recently platelet transfusion was reserved for the management of substantial haemorrhages. Technical advances have enabled fresh platelets to be more readily available than formerly, so that prophylactic platelet-replacement therapy is now possible. When sufficient quantities of platelet concentrates are available effective haemostasis can be maintained even in patients with very low platelet counts. Haemorrhages that would formerly have been fatal can now be averted, and operations can be carried out that would have been too hazardous without the concentrates.

Platelet transfusions are especially beneficial to patients in whom thrombocytopenia is the result of bone marrow failure. In aplastic anaemia haemorrhage is one of the main causes of death. ${ }^{1}$ Some patients suffering from this disease eventually recover their bone marrow function either spontaneously or as a result of treatment, and for them prophylactic platelet transfusions can successfully prevent bleeding attacks over long periods. ${ }^{2-5}$ Platelet transfusion can also be an essential support in the management of acute leukaemia. It helps to prevent haemorrhage both during periods of relapse and during $\stackrel{\odot}{\circ}$ the course of treatment with multiple chemotherapeutic. agents, many of which themselves cause severe depression of $\overrightarrow{\bar{N}}$ bone marrow. ${ }^{2-4}$ Similarly, patients with generalized malignant $\overrightarrow{0}$ disease can be treated with more intensive radiotherapy or $\underline{\bar{O}}$ chemotherapy if platelet transfusions are available to cover $\frac{\bar{\rho}}{\bar{\sigma}}$ the inevitable bone marrow depression.

In the thrombocytopenias associated with adequate or $\%$ increased numbers of megakaryocytes in the bone marrow, $\vec{\circ}$ when the platelet deficiency is presumed to result from their $\overrightarrow{\vec{\omega}}$ destruction by platelet antibodies (as in idiopathic thrombo- ${ }^{\omega}$ cytopenic purpura and the drug-induced immune thrombo- $\frac{5}{3}$ cytopenias), platelet transfusions are of less benefit to the patient. This is because the infused platelets are rapidly of removed from the circulation. However, a transient rise in numbers of platelets does follow transfusion of them in these patients, and this may be valuable in the management of $\frac{}{\triangle}$ potentially fatal haemorrhage or to allow a surgical operation $N$ to be done.

Platelets can be administered in the form of fresh plasma or as a concentrate. Dangerous spontaneous bleeding is rarely seen $^{6}$ if the platelet count is greater than $20,000 / \mathrm{mm}^{3}$. $\vec{\varphi}$ To raise adequately the platelet count in adults with a thrombo-.cytopenia of $10,000 / \mathrm{mm}^{3}$ or less platelets obtained from five blood donations are required. To maintain effective platelet levels in these adult patients requires the transfusion of platelets prepared from ten donors twice a week. Thus $\frac{\mathscr{Q}}{\Phi}$ platelet transfusions make expensive demands on the blood $\cong$ transfusion service. Plasmapheresis, at present available at a $\overrightarrow{\vec{O}}$ few centres, can economize in the provision of large quantities of platelets required for prophylactic transfusions. But unfortunately platelets do not store well under usual blood bank conditions, and they should preferably be used within six hours of their collection.

Even if the problem of supply is solved the effectiveness of long-term treatment with platelets is limited by iso- $\delta$ immunization. Many platelet antigens have been identified. ${ }^{7} 8$ Some appear to be restricted to platelets, while others are $\frac{}{3}$ shared with the leucocytes. Platelets almost certainly carry o antigens identifical to the histocompatibility (HL-A) antigens, $D$ but whether or not they share red cell antigens remains controversial. ${ }^{9-11}$ As platelet-rich plasma and platelet con- $N$ centrates are usually contaminated by red cells it is the practice to use, if possible, platelets prepared from donors of the same $\mathrm{ABO}$ and rhesus group as the recipient.

A practical platelet typing technique has yet to be developed, $\stackrel{\mathbb{\infty}}{\stackrel{\oplus}{+}}$ and selection of donors compatible with respect to platelet 0 antigens is not possible. Fortunately, in comparison with redo्? cells the antigenicity of platelets is low, and no naturally occurr- $\stackrel{\odot}{\odot}$ ing antibodies have been detected. However, repeated trans- $\stackrel{\mathbb{Q}}{\Omega}$ fusions of platelets prepared from a variety of donors over long periods invariably result in isoimmunization, with pro-8 gressively less satisfactory therapeutic responses. ${ }^{510}$ The HL-A antigens seem to be of primary importance. But a을 recent report ${ }^{5}$ has shown that, in patients who had become $?$ refractory to platelets from random donors, platelets obtained from siblings and matched for the main HL-A antigens have continually provided excellent clinical responses. Allergic and 